\title{
Subject Index to Volume 24
}

Acidemia, isovaleric, glycine supplement treatment, 9

Adenine nucleotide, catabolism, during gestation, human trophoblast, 373

Adenosine, trophobastic production, during accelerated adenine nucleotide catabolism, 373

Adenosine triphosphate, changes, hemorrhagic shock, neonatal dog, 438

Adenylosuccinase, deficiency, positron emission tomography, 238

Adipose tissue, abnormalities, detection, topical magnetic resonance spectroscopy, cystic fibrosis, 243

Adrenal gland, chromaffin cell development, nutritional influences, rat, 583

Age

pituitary protein 7B2 plasma levels and, 194

renal response to atrial natriuretic peptide and, young and adult rats, 333

$\beta$-Agonist, combined effect, corticosteroid, thyroid hormones, and, fetal lamb lung, 166

$\beta$-2-Agonist, hepatic glycogen metabolism and, fetal lamb, 330

Airway, surfactant protein content, effect of hyperoxia, rat, 568

Alcohol, prenatal exposure, effect on neonatal sleep cycling and arousal, 101

Alimentation, galactose, intrahepatic metabolic effects, neonatal rat, 302

Alkalosis, respiratory, thromboxane-induced pulmonary hypertension and, piglet, 558

Allergy

cow's milk, antigen absorption by jejunal epithelium, 197

immunoglobulin $\mathrm{E}$ receptor bearing lymphocytes and, 254

Alveolar fluid, pulmonary, calcium increase, neonatal lamb, 322

American Society of Pediatric Nephrology, position paper, 657

Amniocyte, sedimentation analysis, prenatal diagnosis, Zellweger syndrome, 63

Antibody, monoclonal, to group B Streptococcus, type II, mouse, 68

Antigen, absorption, jejunal epithelium, cow's milk allergy, 197

Apolipoprotein $\mathrm{A}_{1}$, lecithin-cholesterol acyl transferase activity and, premature neonates, 191

Apolipoprotein A-I, detection, dyslipoproteinemic family, 222

Arginine vasopressin

cord plasma, asphyxia indicator, 490

hyperosmolality and, ovine fetus, 318

Arousal, oxygen-carbon dioxide interaction, hypoxemia, neonatal lamb, 28

Asphyxia

brain prostanoids after, cerebrospinal fluid, piglet, 229

indices, cord plasma, vasopressin, and erythropoietin as, 490

Atrial natriuretic factor, hyperosmolality and, ovine fetus, 318

Atrial natriuretic peptide, renal effects, young and adult rats, 333
Baroreceptor, afferents, stimulus-response characteristics, fetal and newborn sheep, 342

Bile acid, maximum secretory rate, taurine influence, neonatal guinea pig, 34

Bilirubin, cerebellopathy, tin-protoporphyrin effect, neonatal rat, 209

Biotin, deficiency, fatty acid composition and, weanling rats, 396

Birth, premature, effect on hemoglobin switching, sheep, 688

Blood

fetal, erythroid colonies, growth patterns, 605

hydroxyeicosatetraenoic acid production, adults versus neonates, 563

\section{Blood flow}

cerebral

assessment methods compared, neonates, 461

carbon dioxide reactivity, premature infants, 175

Doppler velocimetry and, neonatal lamb, 423

hyperviscosity and, neonatal piglet, 486 indomethacin effect, pressure ventilation, neonatal pig, 59

intestinal collateral, characterization, piglet, 473

intracranial arterial velocity, Doppler velocimetry and, neonatal lamb, 423

pulmonary, indomethacin effect, fetal lamb, 696

regional cerebral, autoradiographic determination, hypoglycemia, neonatal dog, 41

velocity, ophthalmic artery, term and preterm neonates, 613

velocity waveforms, fetal well-being and intrauterine growth retardation, 609

Body volume, measurement, acoustic plethysmography, infant pig, 85

Bone marrow, transplantation, neurological effects, canine lysosomal storage disease, 347

Bone mineral content, total body calcium reflected by, neonatal piglet, 693

Brain

insulin receptor, heterogeneity, fetus, 683

maturation

prenatal dexamethasone and, rat, 465

thyroid hormone deficiency and, fetal rat, 588

Breast-feeding, antioxidant properties of human colostrum, 14

Bronchiolitis, respiratory syncytial virus, leukotriene release during, 504

Bronchoalveolar lavage, high frequency flowinterrupter performance after, rabbit, 203

Bronchopulmonary dysplasia

evolution, diffuse alveolar damage and, baboon, 357

pulmonary hypertension in, nifedipine effect, infants, 186

\section{Calcium}

cell levels, nasal epithelium, cystic fibrosis, 79 pulmonary alveolar, neonatal lamb, 322

total body, bone mineral content and, neonatal piglet, 693

transport

during development, vitamin D role, suckling rat, 338

maturation, cardiac sarcoplasmic reticulum, sheep, 639

Captopril, distribution of left ventricular output and, ventricular septal defect, lamb, 499

Carbon dioxide

elimination, during liquid ventilation, cat, 291

reactivity, cerebral circulation, premature infants, 175

Cardiac output, regulation with controlled heart rate, neonatal lamb, 577

Cardiogenesis, trichloroethylene effect, chick embryo, 740

Carnitine palmitoyl transferase, deficiency, hepatic and muscular presentations, 308

Carotid, baroreceptor activity, fetal and newborn sheep, 342

$\beta$-Casomorphin, intestinal secretion of electrolytes and, rabbit, 751

Catecholamine, during nitroprusside-induced hypotension, pregnant and fetal sheep, 73

Celiac disease, gliadin peptide digestion and, fetal rat intestine and cultured celiac mucosa, 233

Central nervous system, infection, antibacterial agents, neurologic toxicity, rat brain cell culture, 367

Cerebrospinal fluid, prostanoids, postasphyxial increases, piglet, 229

Chediak-Higashi syndrome, polymorphonuclear lymphocytes in, fluorescent cytometric analysis, infant, 673

Chlorine, transport, pancreas, cystic fibrosis model, rat, 427

Chromaffin cell, adrenal, development, nutritional influences, rat, 583

Circulation: see Blood flow

Colon, sodium transport mechanisms, suckling and weanling rat, 46

Colony-forming units, granulocyte/macrophage, role in ontogeny of human hemopoiesis, neonate, 701

Colostrum, human, antioxidant effects, 14

Contamination, microbial, role of bronchoalveolar macrophages, human and rabbit newborn, 285

Cord blood

hydroxyeicosatetraenoic acid production, 563

immunoglobulin $\mathrm{G}$ fraction, opsonic and protective activity against group $B$ streptococcus, 628

Corticosteroid, combined effect, thyroid hormones, $\beta$-agonist, and, fetal lamb lung, 166

Corticosterone, plasma concentrations, kinetics, infant rat, 595

Cysteine, hepatic metallothionein as source of, first year of life, 326

Cystic fibrosis 
abnormal chlorine transport, pancreatic acinar cells, rat, 427

adipose tissue abnormalities, topical magnetic resonance spectroscopy, 243

essential fatty acid deficiency, role of caloric supplementation, 353

nasal epithelium, cell calcium levels, 79

red blood cells, rubidium transport pathways, 495

Cytochrome $c$ oxidase, deficiency, muscular, mitochondrial electron-transfer chain defects, 447

Cytometry, fluorescent, polymorphonuclear leukocyte analysis, Chediak-Higashi syndrome, infant, 673

5'-Deiodinase, developmental changes, fetal rat brain, 588

Dexamethasone, prenatal, ornithine decarboxylase activity development and, brain tissue, rat, 465

Diabetes, surfactant apoprotein concentration, amniotic fluid, 728

Diaphragm, gavage feeding effect, preterm infants, 55

Dimethylthiourea, effect on group B Streptococcus-induced pulmonary hypertension, piglet, 735

2,3-Diphosphoglycerate, postnatal rise, glucose perfusion and, neonatal lamb, 470

Dopamine receptor, pulmonary, maturation,

Drugs pulmonary artery, rabbit, 160

antibiotic, neurologic toxicity, dissociated brain cell cultures, neonatal rat, 367

antimicrobial, neurologic toxicity, dissociated brain cell cultures, neonatal rat, 367

Dyslipoproteinemia, apolipoprotein A-I mutant and, 222

Electrical impedance, cerebral blood flow measurement, compared with

${ }^{133}$ Xenon clearance, neonates, 461

Electrolyte

intestinal secretion, $\beta$-casomorphins and, rabbit, 751

red blood cell, changes, hemorrhagic shock, neonatal dog, 438

Electron spin resonance, reacting sulfhydryl groups, neonatal erythrocytes, 391

Embryo, heart, trichoroethylene-associated teratogenicity, chick, 740

Enterocolitis, inflammatory response, Hirschsprung's disease, mouse, 152

Enterocyte, small intestinal, Salmonella typhimurium adherence, effect of postnatal development, rat, 508

Enzyme replacement

lysosomal storage disorder and, cultured skeletal muscle, infant, 90

prolidase deficiency and, 709

Epithelium, nasal, cell calcium levels, cystic fibrosis, 79

Erythrocyte: see also Red blood cells

incorporation of ingested 58-iron, infants, 20

intracellular vesicle formation, hyposplenism and, neonate, 703

prolidase, in situ activation, 709

Erythropoietin, cord plasma, asphyxia indicator, 490

Estrogen, effect on bile salt sulfotransferase activities, rat, 247

European Society for Paediatric Endocrinology, abstracts of annual meeting, 517
European Society for Paediatric Gastroenterology and Nutrition (ESPGAN), abstracts of annual meeting, 405

European Society for Paediatric Infectious Diseases, abstracts of annual meeting, 653

European Society for Pediatric Research, plenary lectures and abstracts, 258

Exercise, cardiorespiratory response, after Fontan procuedure for tricuspid atresia, 1

Fatty acid

composition abnormalities, biotin-deficient rats, 396

essential, deficiency, caloric supplementation, cystic fibrosis, 353

polyunsaturated, abnormalities, cystic fibrosis, topical magnetic resonance spectroscopy, 243

Ferritin, serum concentration, erythrocyte

Fetus incorporation of iron and, infants, 20

accelerated growth, after selective uterine artery ligation and, rat, 384

baroreceptor afferents, stimulus-response characteristics, sheep, 342

blood flow, growth retardation and, 609

brain, insulin receptor, heterogeneity, 683

developmental changes, $5^{\prime}$-deiodinase and thyroid hormones, rat brain, 588

erythroid colonies, growth patterns, 605

glycogen metabolism, hepatic, effects of $\beta$ 2 agonist, lamb, 330

growth acceleration, human placental lactogen and, rat, 663

growth-retarded, precocial neural function, lamb, 600

hyperosmolality, atrial natriuretic factor and arginine vasopressin response, sheep, 318

hypotension, plasma vasopressin, renin, and catecholamines during, sheep, 73

insulin deficiency, uteroplacental glucose uptake and, sheep, 312

lung

chronic glucose infusion and, lamb, 171

corticosteroid, thyroid hormones and $\beta$ agonist effect, lamb, 166

maternal hypoxia and, rat, 156

ontogeny of pulmonary protein phosphatases, rat, 25

stability, inositol and glucocorticoid role, rabbit, 617

response to increased oxygen tension, indomethacin effect, lamb, 696

Fluorocarbon, ventilation, $\mathrm{CO}_{2}$ elimination during, cat, 291

Fontan procedure, tricuspid atresia, noninvasive exercise testing, 1

Galactose, alimentation, intrahepatic metabolic effects, neonatal rat, 302

Gas exchange, during high frequency flowinterruption, after bronchoalveolar lavage, rabbit, 203

Gastroesophageal reflux, nonnutritive sucking and, infants, 38

Gavage feeding, see Nutrition

Glucocorticoid, lung stability and, fetal rabbit, 617

Glucose

chronic infusion, $\beta$-receptor binding and, fetal lamb lung, 171

deprivation, adenine nucleotide metabo- lism and, during gestation, 373

intolerance, congenital generalized lipodystrophy, 668

perfusion, effect on 2,3-diphosphoglycerate, neonatal lamb, 470

regional brain utilization, adenylosuccinase deficiency, 238

uteroplacental uptake, fetal insulin concentration and, sheep, 312

Glycine, isovaleric acidemia treated by, 9

Glycogen

hepatic

after galactose alimentation, neonatal rat, 302

metabolism, effects of $\beta-2$ agonist, fetal lamb, 330

Glycogenosis type II, enzyme supplementation and, cultured skeletal muscle, infant, 90

Growth and development

accelerated fetal growth, selective uterine artery ligation and, rat, 384

adrenal chromaffin cell, nutritional influences, rat, 583

calcium transport

cardiac sarcoplasmic reticulum, age-related changes, sheep, 639

vitamin D role, suckling rat, 338

chronotropic response to $\alpha$-stimulation, maturational changes, dog, 50

corticosterone role, infant rat, 595

5 '-deiodinase and thyroid hormones, fetal rat brain, 588

fetal, human placental lactogen and, rat, 663

growth hormone-releasing hormone treatment, growth hormone-deficient children, 145

intestinal circulation, piglet, 473

maternal hypoxia, fetal lung growth and, rat, 156

mental health predictors, hypothyroid children, 6

pituitary protein $7 \mathrm{~B} 2$, changes in plasma levels during childhood, 194

postnatal catch-up growth, after intrauterine growth retardation, 380

pulmonary dopamine receptor maturation, pulmonary artery, rabbit, 160

sodium transport, colonic, mechanisms during ontogeny, rat, 46

Growth hormone, deficiency, pancreatic polypeptide levels and, 574

Growth hormone-releasing hormone, growth rate increased by, growth hormonedeficient children, 145

Growth retardation

intrauterine

blood flow velocity waveforms and, 609

cerebral tolerance of hypoxia and guinea pig, 633

postnatal catch-up growth after, 380

neonatal hypoglycemia and, cerebral metabolic response, dog, 649

precocial neural function and, fetal lamb, 600

Haemophilus influenzae type $\mathrm{b}$, antibodies, subclass distribution, 180

Heart, sarcoplasmic reticulum, maturation of calcium transport, sheep, 639

Heart defect, trichloroethylene and, chick embryo, 740

Heart rate

controlled, regulation of cardiac output with, neonatal lamb, 577 
interaction with respiration, neonate, 745

Hemoglobin, switching, premature parturition and, sheep, 688

Hemopoiesis, ontogeny, role of granulocyte/ macrophage colony-forming units, neonate, 701

Hirschsprung's disease, enterocolitis immunopathology, mouse, 152

Howland Award

acceptance, 514

presentation, 512

Human purine and pyrimidine metabolism, VI International Symposium, abstracts, 111

4-Hydroxybutyric aciduria, succinic semialdehyde dehydrogenase measurement, cultured human lymphoblasts, 455

12-Hydroxyeicosatetraenoic acid, impaired production, neonate, 563

Hyperbilirubinemia, tin-protoporphyrin effect, neonatal rat, 209

Hypercarbia, brain blood flow during, Doppler velocimetry, neonatal lamb, 423

Hyperosmolality, atrial natriuretic factor and arginine vasopressin response, ovine fetus, 318

Hyperoxia, surfactant protein and, rat, 568

Hypertension

pulmonary

group B Streptococcus-induced, dimethylthiourea effect, piglet, 735

in bronchopulmonary dysplasia, nifedipine effect, infants, 186

respiratory alkalosis and, piglet, 558

Hyperviscosity, cerebral blood flow and, neonatal piglet, 486

Hypervolemia, atrial natriuretic factor and arginine vasopressin response, ovine fetus, 318

Hypocarbia, brain blood flow during, Doppler velocimetry, neonatal lamb, 423

Hypoglycemia

cerebral metabolic response, growth-retarded neonatal dog, 649

regional cerebral blood flow during, neonatal dog, 41

Hyposplenism, intracellular vesicle formation and, neonatal erythrocyte, 703

Hypotension, fetal and maternal, plasma vasopressin, renin, and catecholamines during, sheep, 73

Hypothyroidism, mental health, predictors of eventual outcome, 6

Hypotonia, magnetic resonance spectroscopic study, rickets, 713

Hypoxanthine, cord plasma, asphyxia indicator, 490

Hypoxemia

arousal response, neonatal lamb, 28

arterial, group B Streptococcus-induced, dimethylthiourea effect, piglet, 735

Hypoxia, cerebral tolerance, growth retardation and, neonatal guinea pig, 633

Ileum, electrolyte secretion, $\beta$-casomorphins and, rabbit, 751

Immunization, Haemophilus influenzae type $b$, immunoglobulin $G$ subclass response, 180

Immunodeficiency, combined, clonal $\mathrm{T}$ cell populations in, 622

Immunoglobulin $\mathrm{G}$, subclass response, Haemophilus influenzae type $\mathrm{b}$ immunization, 180

Indomethacin

carbon dioxide reactivity of cerebral cir- culation and, premature infants, 175

cerebral blood flow restricted by, during pressure ventilation, neonatal pig, 59

intrauterine exposure, renal impairment and, preterm neonate, 644

pulmonary circulation and, fetal lamb, 696

Infant: see also Neonate

body volume, acoustic plethysmographic determination, pig, 85

bronchopulmonary dysplasia, pulmonary hypertension, nifedipine effect, 186

Chediak-Higashi syndrome, C3bi receptor expression, infant, 673

erythrocyte incorporation of ingested 58iron, 20

lysomal storage disorder, correction, cultured skeletal muscle, 90

nonnutritive sucking, gastroesophageal reflux and, 38

ocular compression test, maturation effect on heart rate response, 477

plasma concentrations of corticosterone, kinetics, rat, 595

premature

carbon dioxide reactivity of cerebral circulation, 175

diaphragm function after gavage feeding, 55

serum lecithin-cholesterol acyl transferase and apolipoprotein $A_{1}$ levels, 191

protein metabolism, after reduced protein formula, 297

Zellweger syndrome, diagnosis by rectal biopsy, 723

Infant formula, reduced protein, protein metabolism during weaning and, 297

Infection, central nervous system, antibacterial agents, neurologic toxicity, rat brain cell culture, 367

Inflammation, antioxidant effects of human colostrum, 14

Inositol, lung stability and, fetal rabbit, 617

Insulin

deficiency, fetus, uteroplacental glucose uptake and, sheep, 312

receptor, heterogeneity, fetal brain, 683

resistance, congenital generalized lipodystrophy, 668

Insulin-like growth factor 1, growth-promoting activity, after intrauterine growth retardation, 380

Interleukins, general information, 549

Intestine

celiac, digestion of gliadin peptides, 233

retinol uptake by, suckling and adult rats, 481

Iron, stable isotope, ingestion, infants, 20

Jaundice, tin-protoporphyrin effect, neonatal rat, 209

Jejunum, epithelium, antigen absorption by, cow's milk allergy, 197

Kidney

atrial natriuretic peptide infusion and, young and adult rats, 333

functional impairment, intrauterine indomethacin exposure and, preterm neonate, 644

Lactogen, human placental, fetal growth accelerated by, rat, 663

Leucine, glycine supplement and, isovaleric acidemia, 9

Leukocyte, polymorphonuclear, fluorescent cytometric analysis, Chediak-Higashi syndrome, infant, 673
Leukotriene, release in respiratory tract, during respiratory syncytial virus infection, 504

Lipid, fluidity, membrane, red blood cells, adults and newborns, 433

Lipodystrophy, congenital generalized, insulin resistance in boy with, 668

Lung

alveolar fluid, calcium increase, neonatal lamb, 322

corticosteroid, thyroid hormones, and $\beta$ agonist effect, fetal lamb, 166

diffuse alveolar damage, bronchopulmonary dysplasia, baboon, 357

macrophages, anti-Candidia activity, human and rabbit newborn, 285

maternal hypoxia and, fetal rat, 156

maturation, prenatal dexamethasone and, rat, 465

ontogeny, pulmonary protein phosphatases, fetal and neonatal rat, 25

$\beta$-receptor binding, chronic glucose infusion and, fetal lamb, 171

stability, inositol and glucocorticoid role, fetal rabbit, 617

Lymphocyte, immunoglobulin E receptorbearing, allergic and nonallergic children, 254

Lymphotoxin, production, neonatal mononuclear cells, 717

Lysosomal storage disease, bone marrow transplantation and, neurological effects, $\operatorname{dog}, 347$

Macrophage

bronchoalveolar, microbicidal function, human and rabbit newborn, 285

monocyte-derived, phagocytosis-associated functions, neonate, 213

Magnetic resonance spectroscopy, hypotonia of rickets, 713

Malnutrition, essential fatty acid deficiency, caloric supplementation, cystic fibrosis, 353

Marijuana, prenatal exposure, effect on neonatal sleep cycling and arousal, 10

Maturation

heart rate response as a function of, ocular compression test, premature infants, 477

neurologic, growth-retarded fetal lamb, 600

Megacolon, congenital, enterocolitis immunopathology, mouse, 152

Membrane fluidity, abnormal, neonatal polymorphonuclear leukocytes, 442

Mental health, hypothyroid children, predictors of eventual outcome, 6

Metabolism

fatty acid, biotin deficiency and, rat, 396

lysosomal storage disease, neurological effects of bone marrow transplantation, dog, 347

Metallothionein, hepatic, as zinc and cysteine source, 326

Milk, cow's, allergy, antigen absorption by jejunal epithelium of children with, 197

Mitochondria, myopathy, electron-transfer chain defects, 447

Mononuclear cells, neonatal, production of lymphotoxin and tumor necrosis factor by, 717

Mucopolysaccharidosis I, bone marrow transplantation and, neurological effects, $\operatorname{dog}, 347$

Mucosa, rectal, peroxisomes, Zellweger syn- 
drome and, infant, 723

Muscle, cytochrome $c$ oxidase deficiency, electron-transfer chain defects, 447

Necrotizing enterocolitis, pathogenesis, intestinal circulation, characterization, piglet, 473

Neonate

arousal response to hypoxemia, lamb, 28

asphyxia, brain prostanoids after, piglet, 229

baroreceptor afferents, stimulus-response characteristics, sheep, 342

bile acid maximum secretory rate, taurine influence, guinea pig, 34

blood flow velocity, ophthalmic artery, term and preterm infants, 613

bone mineral content, total body calcium reflected by, piglet, 693

bronchoalveolar macrophages, microbicidal function, 285

cardiac output, regulation with controlled heart rate, lamb, 577

cerebral blood flow assessment, electrical impedance versus ${ }^{133}$ Xenon clearance, 461

chromaffin cell development, nutritional influences, rat, 583

chronotropic effects of $\alpha$-stimulation, maturation and, $\operatorname{dog}, 50$

enteric galactose alimentation, intrahepatic metabolic effects, rat, 302

erythrocyte, intracellular vesicle formation, 703

erythrocyte properties, reacting sulhydryl groups and, 391

glucose perfusion, effect on 2,3-diphosphoglycerate concentration, lamb, 470

heart rate-respiration interaction, 745

hemopoiesis ontogeny, role of granulocyte/macrophage colony-forming units, neonate, 701

hemorrhagic shock, red blood cell electrolyte changes, dog, 438

hyperbilirubinemia, tin-protoporphyrin effect, rat, 209

hyperviscosity, cerebral blood flow and oxygen metabolism, piglet, 486

hypoglycemia

cerebral metabolic response, growth-retarded dog, 649

regional cerebral blood flow during, dog, 4

hypoxia, cerebral tolerance, growth retardation and, guinea pig, 633

impaired 12-hydroxyeicosatetraenoic acid production, 563

lung, ontogeny of pulmonary protein phosphatases, rat, 25

monocyte-derived macrophages, phagocytosis-associated functions, 213

mononuclear cells, production of lymphotoxin and tumor necrosis factor by, 717

polymorphonuclear leukocytes, abnormal membrane properties, 442

positive pressure ventilation, indomethacin effect, pig, 59

pulmonary alveolar fluid, calcium increase, lamb, 322

sleep cycling and arousal, prenatal alcohol and marijuana effect, 101

Nephrology, current status and future needs, American Society of Pediatric Nephrology, position paper, 657

Nervous system, vagal cholinergic, growth hormone deficiency and, 574
Nifedipine, hemodynamic effects, pulmonary hypertension in bronchopulmonary dysplasia, infants , 186

Nutrition

caloric supplementation, essential fatty acid deficiency, cystic fibrosis, 353

chromaffin cell development and, adrenal gland, rat, 583

gavage feeding, diaphragm function after, preterm infants, 55

reduced protein formula, protein metabolism during weaning and, 297

Ocular compression test, heart rate response, maturation effect, infant, 477

Ophthalmic artery, blood flow velocity, term and preterm neonates, 613

Ornithine decarboxylase, development, prenatal dexamethasone and, brain tissue, rat, 465

Oxygen

deprivation, adenine nucleotide metabolism and, during gestation, 373

metabolism, hyperviscosity and, neonatal piglet, 486

Pacifier, effect on gastroesophageal reflux, infants, 38

Pancreas, abnormal chlorine transport, cystic fibrosis model, rat, 427

Peptide, gliadin, celiac intestine and digestion of, 233

Peroxisomes, rectal mucosa, Zellweger syndrome, infant, 723

Pituitary protein 7B2, plasma levels, changes during childhood, 194

Plethysmography, acoustic, body volume and fat-free mass determinations by, infant pig, 85

Polymorphonuclear leukocyte

human colostrum effect, 14

neonatal, abnormal membrane properties, 442

Polypeptide, pancreatic, growth hormone deficiency and, 574

Pregnancy

adenine nucleotide catabolism during, human trophoblast, 373

alcohol and marijuana use during, effect on neonatal sleep cycling and arousal, 101

diabetic, surfactant apoprotein in, 728

human placental lactogen administration, effect on fetal growth, rat, 663

hypotension, plasma vasopressin, renin, and catecholamines during, sheep, 73

maternal hypoxia, lung growth and, fetal rat 156

Prematurity: see also Infant, premature

diaphragm function after gavage feeding, 55

hormone-induced, effect on hemoglobin switching, sheep, 688

ocular compression test, heart rate response to, 477

renal impairment, intrauterine indomethacin exposure and, 644

retinopathy, reduced severity, $28 \%$ inspired oxygen, kitten, 106

retinopathy of, ophthalmic blood flow ve locity, 613

Pressure, positive end-expiratory, optimal therapy, acute respiratory failure, 217

Prolidase, human erythrocyte, in situ activation, 709

Prostanoids, postasphyxial increases, cerebrospinal fluid, piglet, 229
Protein

metabolism, during weaning, after reduced protein formula, 297

transport, cow's milk allergy, 197

Protein A, surfactant-associated, hyperoxia and, rat, 568

Protein phosphatase, pulmonary, ontogeny, developing rat lung, 25

Puberty, pituitary protein 7B2 plasma levels and, 194

Pulmonary artery, dopamine-induced relaxation, maturation-related changes, rabbit, 160

Pyruvate dehydrogenase, deficiency, fibroblast detection, 95

Red blood cells

electrolyte changes, neonatal shock, dog, 438

membrane fluidity, adults and newborns, 433

reacting sulfhydryl groups in, neonates, 391

rubidium transport pathways, kinetic parameters, cystic fibrosis, 495

Renin, during nitroprusside-induced hypotension, pregnant and fetal sheep, 73

Respiration, interaction with heart rate, neonate, 745

Respiratory distress syndrome, hyperoxia and surfactant protein, rat, 568

Respiratory failure, acute, optimal positive end-expiratory pressure, 217

Respiratory sinus, arrhythmia, sudden infant death syndrome, 677

Respiratory syncytial virus, respiratory tract infection, leukotriene release during, 504

Reticulum, sarcoplasmic, maturation of calcium transport, sheep, 639

Retinol, intestinal uptake, characteristics and ontogeny, suckling rat, 481

Retinopathy, oxygen-induced, reduced severity, 28\% inspired oxygen, kitten, 106

Rickets, hypotonia, magnetic resonance spectroscopic study, 713

Rubidium, transport pathways, red blood cells, cystic fibrosis, 495

Salmonella typhimurium, adherence to small intestinal enterocytes, postnatal development effect, rat, 508

Sedimentation analysis, prenatal diagnosis, Zellweger syndrome, 63

Shock, hemorrhagic, red blood cell electrolyte changes, dog, 438

Sinus node, chronotropic effects of $\alpha$-stimulation, maturation and, neonatal dog, 50

Skin, fibroblast, pyruvate dehydrogenase deficiency and, 95

Sleep

arousal response, hypoxemia and, neonatal lamb, 28

cycling and arousal, prenatal alcohol and marijuana effect, 101

Small intestine, postnatal development, Salmonella typhimurium adherence and, rat, 508

Sodium, colonic transport, mechanisms, during ontogeny, immature rat, 46

Spectral analysis, respiratory sinus arrhythmia assessed by, sudden infant death syndrome, 677

Spectroscopy, topical magnetic resonance, adipose tissue abnormality detection, cystic fibrosis, 243 
Stimulation, $\alpha$-adrenergic, maturational changes of chronotropic response, dog, 50

Streptococcus

group B

dimethylthiourea effect, pulmonary hypertension induced by, piglet, 735

opsonic and protective activity against, human cord sera, 628

group B, type II, monoclonal antibodies to, mouse, 68

Succinic semialdehyde dehydrogenase, whole cell assay, cultured human lymphoblasts, 455

Sudden infant death syndrome, respiratory sinus arrhythmia, spectral analysis assessment, 677

Sulfotransferase isoenzyme, hepatic bile salt, sexual differentiation, rat, 247

Surfactant, apoprotein, amniotic fluid, diabetic pregnancy, 728

Taurine, bile acid maximum secretory rate influenced by, neonatal guinea pig, 34

$\mathrm{T}$ cell, oligoclonal proliferative disorder, in combined immunodeficiency, 622

Thromboxane, pulmonary hypertension induced by, respiratory alkalosis and, piglet, 558

Thyroid, mental health and, hypothyroid children, 6
Thyroid hormone

combined effect, corticosteroid, $\beta$-agonist, and, fetal lamb lung, 166

developmental changes, fetal rat brain, 588

Tin-protoporphyrin, pharmacological and biological effects, hyperbilirubinemia, neonatal rat, 209

Tomography, positron emission, adenylosuccinase deficiency, 238

Transplantation, bone marrow, neurological effects, lysosomal storage disease, dog, 347

Trichloroethylene, cardiac teratogenicity, chick embryo, 740

Tricuspid, atresia, Fontan procedure, response to exercise after, 1

Trophoblast, adenylate catabolism, early and late gestation, 373

Tumor necrosis factor, production, neonatal mononuclear cells, 717

Ultrasound, Doppler, blood flow velocity, neonatal lamb, 423

Uterine artery, selective ligation, accelerated fetal growth after, rat, 384

Vasoconstriction, pulmonary, respiratory alkalosis and, piglet, 558

Vasopressin, during nitroprusside-induced hypotension, pregnant and fetal sheep,
73

Ventilation

high frequency flow-interruption, gas exchange, after bronchoalveolar lavage, rabbit, 203

liquid, $\mathrm{CO}_{2}$ elimination during, cat, 291

positive pressure, indomethacin effect, neonatal pig, 59

Ventricular septal defect, left ventricular output distribution, captopril effect, lamb, 499

Vitamin A, alcohol, intestinal uptake, suckling rat, 481

Vitamin D, calcium transport stimulated by, suckling rat, 338

Weaning, protein metabolism during, after reduced protein formula, 297

${ }^{133}$ Xenon clearance, cerebral blood flow assessment, compared with electrical impedance, neonates, 461

\section{Zellweger syndrome}

diagnosis by rectal biopsy, infant, 723

prenatal diagnosis, sedimentation method, 63

Zinc, hepatic metallothionein as source of, first year of life, 326

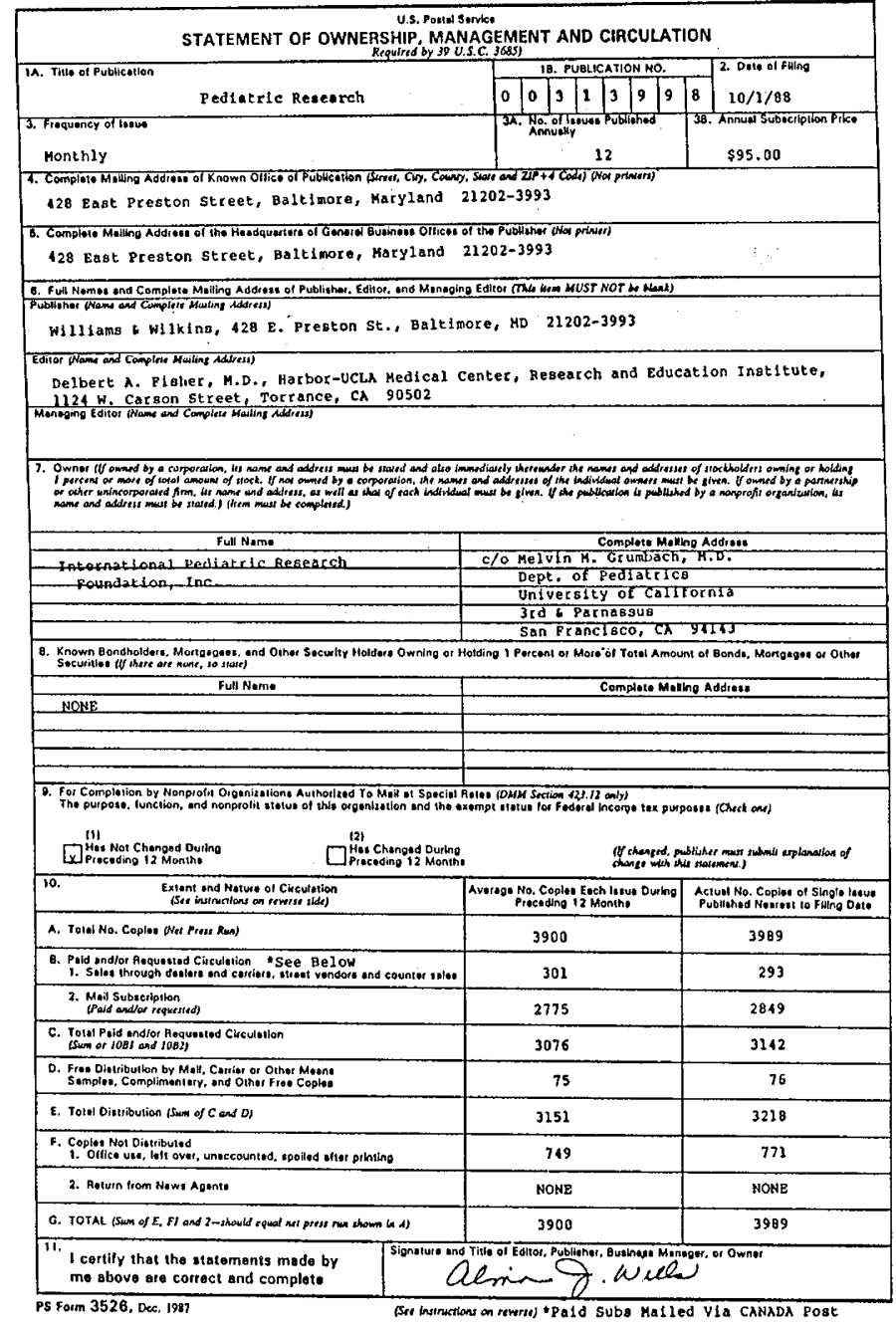

